\title{
Developing collaboration tool for virtual team using UML models
}

Yasmin Makki Mohialden, Huda Abdulaali abdulbaqi, Narjis Mezaal Shati

Computer Science Department, Collage of Science, Mustansiriyah University, Baghdad-Iraq

\begin{tabular}{|c|c|}
\hline Article Info & ABSTRACT \\
\hline Article history: & \multirow{9}{*}{$\begin{array}{l}\text { Computer aided software engineering (CASE) uses the unified modelling } \\
\text { language UML to produce executable code to visualize software architectural } \\
\text { blueprint systems. UML is a standard software modeling language for } \\
\text { specifying, constructing, documenting and visualizing the artifacts of } \\
\text { produced software-intensive systems. However, UML lacks virtual } \\
\text { collaboration regardless of their geographical location. The present work } \\
\text { developed a prototype of web-based CASE tool that enables UML modes to } \\
\text { manage software projects as a virtual collaboration tool. The present } \\
\text { methodology starts with the specification of a set of UML triplets; then, the } \\
\text { tool generates the visual models automatically with high quality SVG graphs. } \\
\text { The system eliminates the manual diagram requirements based on the UML } \\
\text { possibility and supported by three diagrams that involves class and use cases. } \\
\text { The independent scripting language was PHP7 and MYSQL } 8.0 \text { which was } \\
\text { used to save the system data. }\end{array}$} \\
\hline Received Jun 1, 2020 & \\
\hline Revised Nov 29, 2020 & \\
\hline Accepted Jan 12, 2021 & \\
\hline Keywords: & \\
\hline CASE tool & \\
\hline SVG graphs & \\
\hline Unified modeling language & \\
\hline Web-based CASE tool & \\
\hline
\end{tabular}

This is an open access article under the CC BY-SA license.

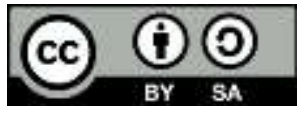

\section{Corresponding Author:}

Yasmin Makki Mohialden

Computer Science Department, Collage of Science

Mustansiriyah University, Baghdad-Iraq

Email: ymmiraq2009@uomustansiriyah.edu.iq

\section{INTRODUCTION}

CASE tools are used to develop high quality applications with defect-free and maintainable software based on end user's perspective [1, 2]. It represents an effective tool for communicating system behaviour which is used for specifying the external visible system behaviou [3-6]. It is partly inspired used by computer aided design CAD tools for hardware products design. The CASE tools are classified into two parts the first is installed CASE tool and the other is web based. The web CASE tools involves many advantages over the desktop based applications. It helps engineering teams to collaborate from different locations all over the world. Also, this software did not required installation process or update $[6,7]$. This feature enable the user to save and administrate the work flow of the large companies. The CASE tools software involves the capability of database accessible by multiple users and multi-computers in real time. This software is feasible and easy due to website uptime [6].

In this paper, a web CASE tools are used to provide less time features and more reliability of user's collaboration. It provides real-time UML design drawing with efficient and good quality [8-12]. The drop and drag facility provides a great power to users. Computer aided software engineering CASE uses the unified modelling language UML to produce executable code to visualize software architectural blueprints systems. UML is a standard software modeling language for specifying, constructing, documenting and visualizing the artifacts of produced software-intensive system. But, UML lacks to virtual collaboration regardless to their geographical location. 
The main contribution of this paper as follow: to develop a prototype of web-based CASE tool which enable UML modes to manage software projects as a virtual collaboration tool. In addition, the present work contains three sections, the second section related works, the third section the proposed method and the section four include Conclusion and future works.

\section{RELATED WORKS}

Planas and Cabot in 2020 proposed a useful lessons to improve the UML modelling process [12-14]. They analyze the modeler's behavior in building UML models and evaluate the modelling tools used for supporting this task. They recommend some changes to enhance the tools effectiveness in order to facilitate the UML learning [15-17]. The researchers observe that the UML turn into better align based on theory and practice applications [18].

Babar et al. In 2020 addresses the UML extension to implement multidimensional modeling. They used the UML class model in DW applications in order to explore in depth the current MD models based on UML extensions [19-22]. They deduced that the current models lack to accuracy and integrity regarding to available properties and features of MD model. The proposed framework of UML extension mechanism able to model all aspects and features of the MD model specifically. Also, the proposed work was simple, precise, comprehensible and precise. The improved model provides a universal method due to UML familiar and acceptable notations that serve the designers and users [22-26]. Cerra et al. In 2018 produced a learning environment based on video and self-assessment combination tools for computer-Aided Design CAD applications. The method developed to analyze the problem and determine the combination effect of the two tools to find out the importance of learning subjects [27-29].

\section{THE PROPOSED METHOD}

\subsection{The used languages in the design}

\subsubsection{PHP}

PHP is general-purpose programing and scripting language which is used to create dynamic interactive websites. The PHP reference implementation continuously produced by the PHP group. The PHP origination was for personal home page applications. Nowadays, it stands for the recursive initialism PHP (hypertext preprocessors). PHP code is executed on web server using PHP interpreter. The implemented code my represents a daemon, a module or as a common gateway Interface executable. Initially, the interpreted and executed PHP code can take any type of data such as binary image data or generated THHP. It forms a whole or part of THHP response. PHP did not have formal specification until 2014, then, is created to be used either in academic site with complete grammar and formal specification, or by enthusiastic programmer.

\subsubsection{HTML5}

HTML5 is a markup language specified with labels, multimedia elements, and user interface. It is used for presenting and structuring World Wide Web content. The HTML5 represents the last version of traditional hypertext markup language which recommended by World Wide Web consortium. The current specification is called the HTML living standard and preserved by major browser vendor consortium (Microsoft, Google, Mozilla, and Apple). Also, it maintained by Web Hypertext Technology Working Group.

\subsubsection{Cascading style sheet}

The cascading style sheet CSS3 can be defined as a style sheet language used to format the layout of web page. It is used to describe the presentation of written documents in markup languages such as HTML. CSS represents the World Wide Web cornerstone technology in addition to HTML and JavaScript. The cascading style sheet mainly designed to enable the separation process in written presentation and content of markup language. It provides more flexibility and control for presentation based on the specification of characteristics. Also, it enables multiple web pages to share formatting separately depending on relevant CSS. The CSS can reduce the repetition and complexity in the structural content in web pages. It facilitate the displayed layout, design and variations using different screen sizes and devices.

\subsubsection{Javascript}

JavaScript is a dynamic programming language compatibles with ECMA Script. It's a high-level language, multi-paradigm program and Just-in-Time compiled. It has curly-bracket syntax, prototype-based object orientation, dynamic typing and first-class function. JavaScript considered one of the World Wide Web core technology. Its similar to HTML and CSS software. It represents an essential part of web 
applications which enables web pages to interact. The client-side page behavior represents a major issue in websites, for that the JavaScript used in all web browsers to execute their engine.

\subsubsection{JQuery}

JQuery is a JavaScript library developed to facilitate HTML DOM (document object model) tree traversal and manipulation. Its open source software utilizing the MIT permissive license. This software able to recognize the actions by using event handling and employ the CSS animation with Asynchronous JavaScript XML (Ajax). Web analysis indicates that this software is widely used in JavaScript library by a large margin. The software usage 3 to 4 times more than any other JavaScript library. JQuery syntax is developed to be effective in document navigation.

\subsubsection{Bootstrap4}

Bootstrap4 is an open-source CSS framework employed to mobile-first front-end web development. I involves CSS and (optionally) JavaScript-based design templates for forms, buttons, typography, navigation and other interface components.

\subsubsection{Database of project}

The database can be defined as an organized collection of information saved in the computer system and have the ability to access and deal with. The database is usually developed by using formal design and modelling techniques in this system a MySQL 8.0, it is a relational database management system RDBMS which is available as an open-source software. Its abbreviation for structured Query Language. The present relational database manages the data based on one or more tables which involves data types. The data organized due to the relations between them and the correlations between the data will help to structure the data. SQL is the program that can extract, modify and create the data form using the relational database. It is also control the users access to the database. The traditional database such as MySQL, SQL and RDBMS correlates with operating system and develop a relational database in the storage system of the computer. It can provide a facility to test the data, allows for network access and create data backup.

\subsubsection{The used severe in the system}

XAMPP 3.2.2 is an open-source cross-platform web server solution stack package. It produces by Apache Friends involving mainly the Apache HTTP Server. The package interpreters for scripts written I the Perl and PHP programs. In general, most of actual web servers apply the same component as XAMPP, for that it became easy to trasition from local test server to a live server.

\subsubsection{The flowchart of the proposed system}

It represents a schematic diagram of the design of the system, which in this paper a n web-based UML CASE for helping a software development team to share the design of the proposed system easily and quickly, even if the team are in different places. This is an important software development step that involves requirements gathering and design analysis, coding/implementation, testing and maintenance. A framework that specifies the steps involvd in every step og software development is referred to as software life cycle (SLC). This framework provides a detailed stepwise guide for the planning, development, deployment and maintenance of the software as shown in Figure 1.

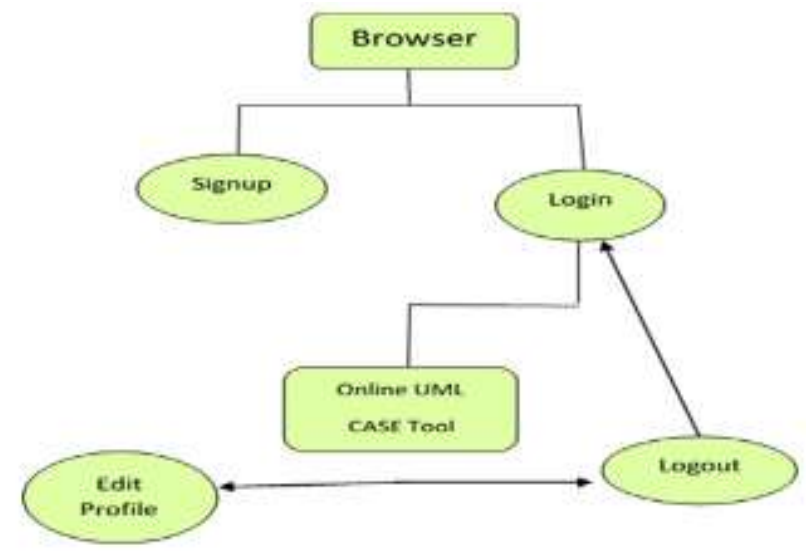

Figure 1. the flowchart of the proposed system 


\subsection{System main pages}

\subsubsection{Login page}

Login is the process of accessing the system in online case individually. UML CASE system provides a user unique name and password to enable the user to access. The user credentials processed by matching the username and the password to identify the access permission and this refer to login (or sign-in or sometimes sign-on).

\subsubsection{Registration web page}

The user registration starting with sign-up function for the first time to provide the credentials to the user access. The registration allows the user to use the website, program or any other system. Registered users provide a sort of credentials to prove their identity and access the system. This process is known as logging in registered user may be granted privileges in addition to granted to unregistered users as shown in Figure 2.

\subsubsection{The online UML CASE tool}

This page contains the main tools of the online UML CASE tool, and contain the properties of tools. As shown in Figure 2. As a dynamic diagram in UML, a UML CASE tool relies on actors and use cases to model the functions of a system. These use cases are a set of functions, services and actions that must be performed by a system. Here, a "system" refers to what is being developed (such as a web site) or operated while the "actors" are entities that are operating within the system under defined roles, Figure 2 describe the UML case tool.

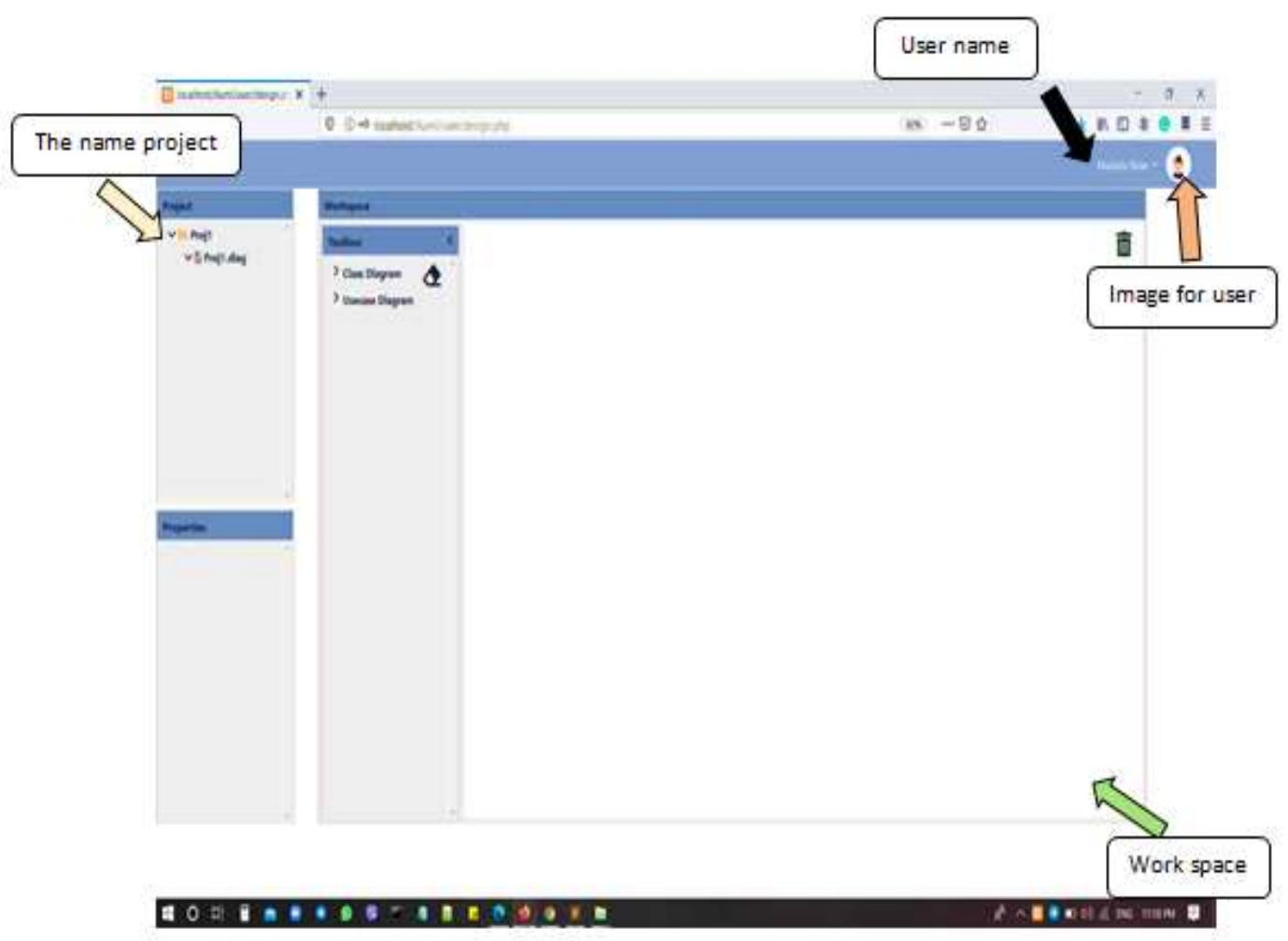

Figure 2. the UML CASE Tool

\subsubsection{Class diagram tool}

This page contains the tools of the class diagram of the online UML CASE tool, and contain the properties of the class diagram tools. It comes with a simple but powerful editor that permits easyand quick creation of class diagrams; the free UML editor can be assessed without time limitation, and comes with no $\mathrm{ad}$; there is also no restriction on the number of shapes and number of diagrams. The users own thst diagrams they created and can use them for non-commercial and personal purposes. As shown in Figure 3 the figure contains the class diagram tool element. 


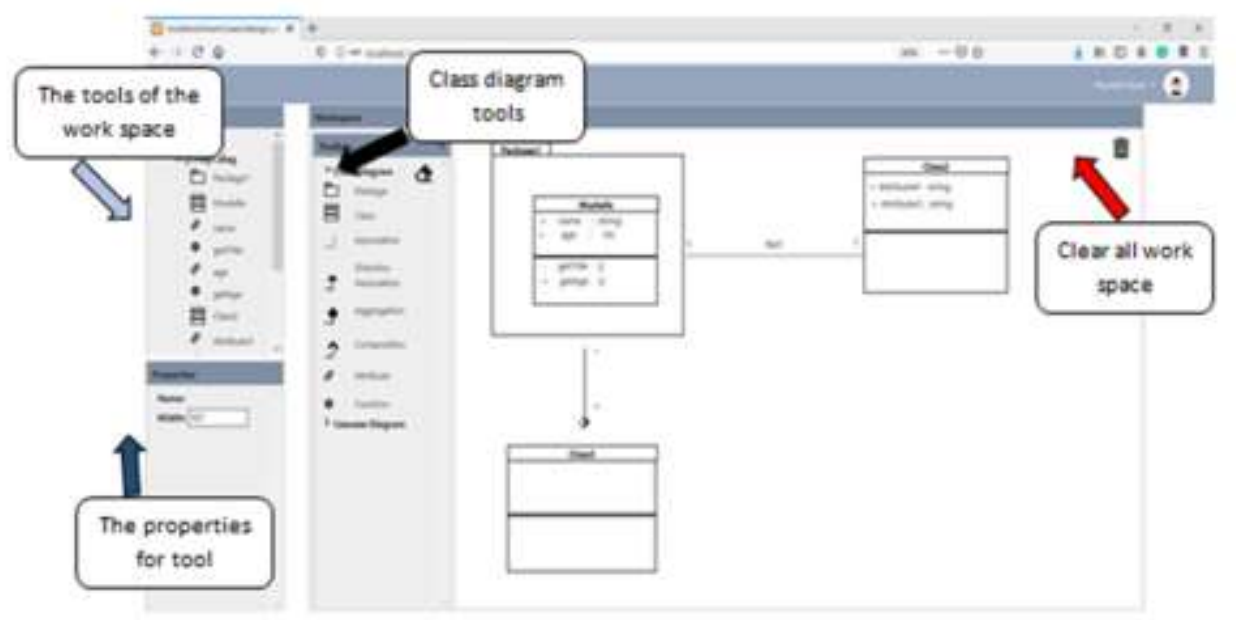

Figure 3. class diagram tool

\subsubsection{Use case diagram tool}

This page contains the tools of the use CASE diagram of the online UML CASE tool, and contain the properties of the user CASE diagram tools. In UML, the use case diagrams are used during the at the requirements phase of software development; the system requirements are normally converted into a diagram format for easy viewing of the functions that must be supported at a glance. Being that a system must have few use cases, the usr is required to draw as my use case diagrams as possible. Use case diagrams, together with the other UML diagrams, such as component diagram, activity diagram, and sequence diagram, aids in viewing the software \& requirements before initiating the programming stage. As shown in Figure 4, the fgure show the use case diagram tool in details.

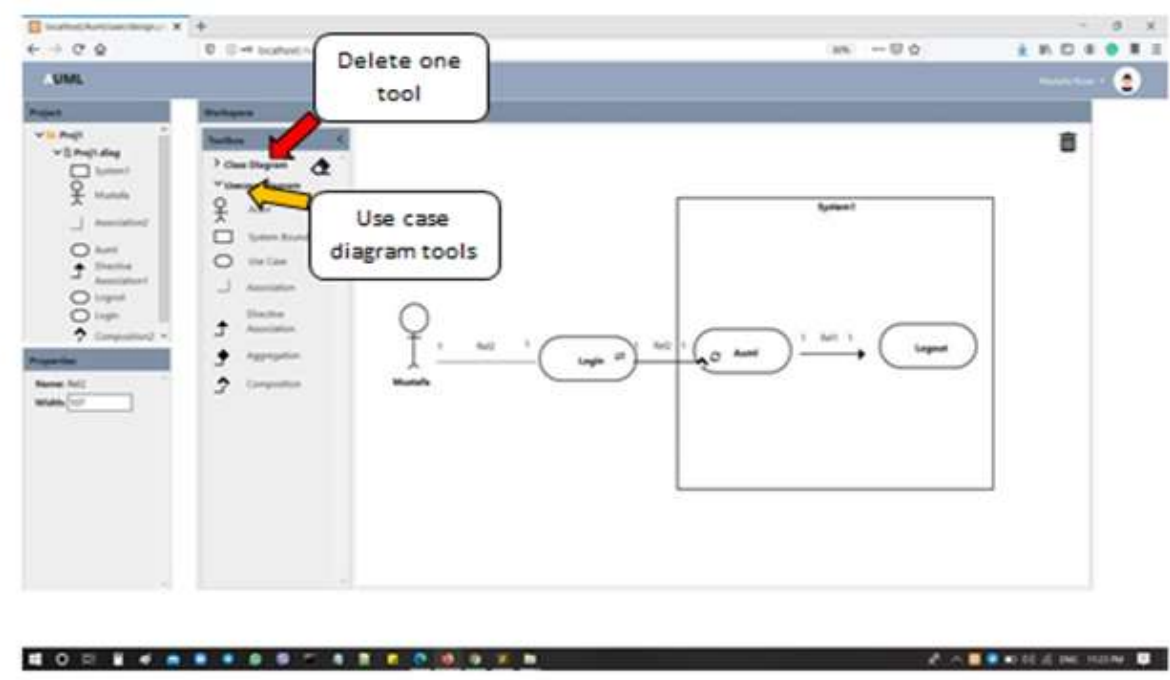

Figure 4. USE CASE diagram example

\subsubsection{Saving diagrams}

In this page each designed diagram can be saved with its associated user profile for editing, retrieving for modifying or or send it for all the collaborative software engineering. This template can be edited at this stage for the creation of the own diagram. The created diagrams can be exported to Word, Powerpoint, Excel, Visio or other document formats. The developed UML diagram tool in this study simplifies the design \& documentation processes and improves the way of collecting, reviewing and implementing responses/feedback from the potential users and stakeholders as shown in Figure 5. 


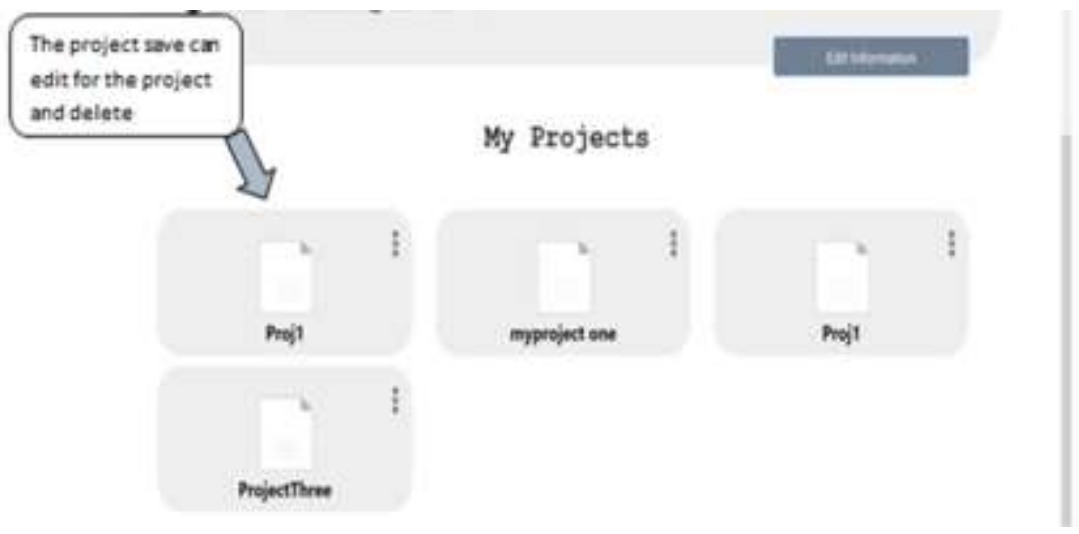

Figure 5. My projects web page to create a new project

\section{CONCLUSION}

In this research, a web-based UML and UML CASE was developed for improving the process of software development in the areas of sharing the design of the proposed system easily and quirkily even when the team members are in different places. The design was correctly built and the system requirements were met as it now works on the web. In this paper, a prototype of web-based CASE tool that enable UML modes to manage software projects as a virtual collaboration tool was developed. The present methodology started with the specification of a set of UML triplets, followed by generation of the visual models automatically with high quality SVG graphs. In the future work, the proposed system will be used to develop AUML (agent UML) diagrams.

\section{ACKNOWLEDGEMENTS}

The Authors thank the Department of Computer Science, College of Science, Mustansiriyah University, Baghdad, Iraq for their support.

\section{REFERENCES}

[1] Bhuvan Unhelkar, "Software Engineering with UML," Auerbach Publications, CRC PRESS, 2018.

[2] "PHP: Hypertext Preprocessor". www.php.net.

[3] Jackson, "PHP gets a formal specification," at last. IT world. IDG, 2015.

[4] "CSS developer guide". Mozilla Developer Network.

[5] Belaidan, S. L. M., "Implementing K-Means Clustering Algorithm in Collaborative Trip Advisory and Planning System," Periodicals of Engineering and Natural Sciences, vol. 7, no. 2, pp. 723-740, 2019, doi: 10.21533/pen.v7i2.461.

[6] Flanagan, David, "JavaScript - The definitive guide (6 Ed.)," 2017.

[7] I. Al Barazanchi, S. A. Hamid, R. A. Abdulrahman, and H. Rasheed, "Automated telemedicine and diagnosis system (ATDS) in diagnosing ailments and prescribing drugs," Period. Eng. Nat. Sci., vol. 7, no. 2, pp. 888-894, 2019, doi ; 10.21533/pen.v7i2.486.

[8] Website Traffic, Statistics and Analytics - Alexa, Www.alexa.com. Retrieved [9] Interview with Kai from the XAMPP project". MySQL AB. Retrieved, 2015.

[9] Planas, E., \& Cabot, J., "How are UML class diagrams built in practice? A usability study of two UML tools: Magicdraw and Papyrus," Computer Standards and Interfaces, vol. 67, 2020, doi: 10.1016/j.csi.2019.103363.

[10] Babar M., Khattak A., Arif F., and Tariq M., "An Improved Framework for Modelling Data Warehouse Systems Using UML Profile,” The International Arab Journal of Information Technology, vol. 17, no. 4, pp. 562-571, 2020, doi: $10.34028 /$ iajit\%2F17\%2F4\%2F15.

[11] P. P. Cerra, J. G. Rodríguez, H. F. Álvarez, and B. B. Parra, "Combining multimedia and self-assessment CAD tools in an interactive web environment to learn engineering drawing," Interactive Learning Environments, vol. 27, no. 2, pp. 1-14, 2018, doi: 10.1080/10494820.2018.1517095.

[12] Zendaoui, F., \& Hidouci, W. K., "Multi-version representation of historical event," Periodicals of Engineering and Natural Sciences, vol. 7, no. 1, pp. 141-147, 2019, doi: 10.21533/pen.v7i1.329.

[13] M. A. Ahmed, R. A. Hasan, A. H. Ali, and M. A. Mohammed, "The classification of the modern arabic poetry using machine learning," TELKOMNIKA (Telecommunication, Computing, Electronics and Control), vol. 17, pp. 2667-2674, 2019, doi: 10.12928/telkomnika.v17i5.12646. 
[14] O. A. Hammood, M. N. M. Kahar, W. A. Hammood, R. A. Hasan, M. A. Mohammed, A. A. Yoob, et al., "An effective transmit packet coding with trust-based relay nodes in VANETs," Bulletin of Electrical Engineering and Informatics (BEEI), vol. 9, pp. 685-697, 2020, doi: 10.11591/eei.v9i2.1653.

[15] R. A. Hasan, M. A. Mohammed, Z. H. Salih, M. A. B. Ameedeen, N. Țăpuş, and M. N. Mohammed, "HSO: A Hybrid Swarm Optimization Algorithm for Reducing Energy Consumption in the Cloudlets," TELKOMNIKA (Telecommunication, Computing, Electronics and Control), vol. 16, pp. 2144-2154, 2018, doi: 10.12928/telkomnika.v16i5.9415.

[16] R. A. Hasan, M. A. Mohammed, N. Țăpuş, and O. A. Hammood, "A comprehensive study: Ant Colony Optimization (ACO) for facility layout problem," in 2017 16th RoEduNet Conference: Networking in Education and Research (RoEduNet), pp. 1-8, 2017 doi: 10.1109/ROEDUNET.2017.8123738.

[17] N. M. Hussien, Y. M. Mohialden, N. T. Ahmed, M. A. Mohammed, and T. Sutikno, "A smart gas leakage monitoring system for use in hospitals," Indonesian Journal of Electrical Engineering and Computer Science (IJEECS), vol. 19, pp. 1048-1054, 2020, doi: 10.11591/ijeecs.v19.i2.pp1048-1054.

[18] H. R. Ibraheem, Z. F. Hussain, S. M. Ali, M. Aljanabi, M. A. Mohammed, and T. Sutikno, "A new model for large dataset dimensionality reduction based on teaching learning-based optimization and logistic regression," TELKOMNIKA (Telecommunication, Computing, Electronics and Control), vol. 18, 2020, doi: 10.12928/telkomnika.v18i3.13764.

[19] M. A. Mohammed and R. A. Hasan, "Particle swarm optimization for facility layout problems FLP-A comprehensive study," in 2017 13th IEEE International Conference on Intelligent Computer Communication and Processing (ICCP), pp. 93-99, 2017, doi: 10.1109/ICCP.2017.8116988.

[20] M. A. Mohammed, R. A. Hasan, M. A. Ahmed, N. Tapus, M. A. Shanan, M. K. Khaleel, et al., "A Focal load balancer based algorithm for task assignment in cloud environment," in 2018 10th International Conference on Electronics, Computers and Artificial Intelligence (ECAI), pp. 1-4, 2018, doi: 10.1109/ECAI.2018.8679043.

[21] M. A. Mohammed, A. A. Kamil, R. A. Hasan, and N. Tapus, "An Effective Context Sensitive Offloading System for Mobile Cloud Environments using Support Value-based Classification," Scalable Computing: Practice and Experience, vol. 20, pp. 687-698, 2019, doi: 10.12694/scpe.v20i4.1570.

[22] M. A. Mohammed, I. A. Mohammed, R. A. Hasan, N. Țăpuş, A. H. Ali, and O. A. Hammood, "Green Energy Sources: Issues and Challenges," in 2019 18th RoEduNet Conference: Networking in Education and Research (RoEduNet), pp. 1-8, 2019, doi: 10.1109/ROEDUNET.2019.8909595.

[23] M. A. Mohammed, Z. H. Salih, N. Țăpuş, and R. A. K. Hasan, "Security and accountability for sharing the data stored in the cloud," in 2016 15th RoEduNet Conference: Networking in Education and Research, pp. 1-5, 2016.

[24] M. A. Mohammed and N. ŢĂPUŞ, "A Novel Approach of Reducing Energy Consumption by Utilizing Enthalpy in Mobile Cloud Computing," Studies in Informatics and Control, vol. 26, pp. 425-434, 2017, doi: 10.1109/RoEduNet.2016.7753201.

[25] N. Q. Mohammed, M. S. Ahmed, M. A. Mohammed, O. A. Hammood, H. A. N. Alshara, and A. A. Kamil, "Comparative Analysis between Solar and Wind Turbine Energy Sources in IoT Based on Economical and Efficiency Considerations," in 2019 22nd International Conference on Control Systems and Computer Science (CSCS), pp. 448-452, 2019, doi: 10.1109/CSCS.2019.00082.

[26] Z. H. Salih, G. T. Hasan, and M. A. Mohammed, "Investigate and analyze the levels of electromagnetic radiations emitted from underground power cables extended in modern cities," in 2017 9th International Conference on Electronics, Computers and Artificial Intelligence (ECAI), 2017, doi: 10.1109/ECAI.2017.8166452.

[27] Z. H. Salih, G. T. Hasan, M. A. Mohammed, M. A. S. Klib, A. H. Ali, and R. A. Ibrahim, "Study the Effect of Integrating the Solar Energy Source on Stability of Electrical Distribution System," in 2019 22nd International Conference on Control Systems and Computer Science (CSCS), pp. 443-447, 2019, doi: 10.1109/CSCS.2019.00081.

[28] S. A.-b. Salman, A.-H. A. Salih, A. H. Ali, M. K. Khaleel, and M. A. Mohammed, "A New Model for Iris Classification Based on Naïve Bayes Grid Parameters Optimization," International Journal of Sciences: Basic and Applied Research (IJSBAR), vol. 40, pp. 150-155, 2018.

[29] N. D. Zaki, N. Y. Hashim, Y. M. Mohialden, M. A. Mohammed, T. Sutikno, and A. H. Ali, "A real-time big data sentiment analysis for iraqi tweets using spark streaming," Bulletin of Electrical Engineering and Informatics (BEEI), vol. 9, pp. 1411-1419, 2020, doi: 11591/eei.v9i4.1897. 